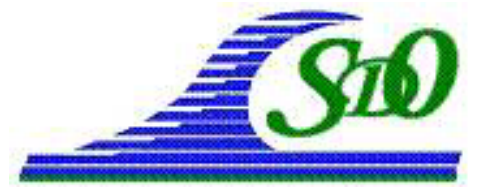

XI ìmes Journées Nationales Génie Côtier - Génie Civil

Les Sables d'Olonne, 22-25 juin 2010

DOI:10.5150/jngcgc.2010.017-T C Editions Paralia CFL

disponible en ligne - http://www.paralia.fr - available online

\title{
Caractérisation des états de mer extrêmes dans les environnements soumis à plusieurs régimes de vagues
}

\author{
Jérôme THIEBOT ${ }^{1}$, Gonéri LE COZANNET ${ }^{1}$, Manuel GARCIN ${ }^{1}$, \\ Rodrigo PEDREROS ${ }^{1}$, Nicolas DESRAMAUT ${ }^{1}$, Etienne DELVALLEE ${ }^{1}$, \\ Ywenn DE LA TORRE ${ }^{1}$, Arnaud BLANGY ${ }^{1}$, Carlos OLIVEROS ${ }^{1}$
}

1. BRGM, RNSC/Risques Côtiers, 3 av. Claude Guillemin, BP 36009, 45060 Orléans Cedex 2, France.

j.thiebot@brgm.fr

\section{Résumé :}

Le calcul des états de mer extrêmes est primordial pour la gestion des risques côtiers. Dans certains environnements météorologiques, les vagues ont des origines variées. Dans l'océan Indien par exemple, les sources de vagues sont nombreuses : houles australes, vagues générées par les vents de mousson, par les alizés ou par les cyclones. Ces différents types de vagues ont des caractéristiques différentes en termes de hauteur significative $\left(\mathrm{H}_{\mathrm{s}}\right)$, de direction, de période mais également en termes de probabilité d'occurrence et donc de période de retour. La présence de plusieurs régimes de vagues rend la détermination des états de mer extrêmes complexe.

L'analyse statistique des états de mer extrêmes se base sur des échantillons d'événements indépendants issus de données de vagues homogènes. Par données de vagues homogènes, on entend des ensembles d'états de mer comparables, c'est à dire associés à des conditions météorologiques similaires. Classiquement, la détermination des ensembles d'état de mer homogènes s'effectue à l'aide de critères simples basés sur les saisons et/ou sur les directions de provenance des vagues. Cette façon de faire n'est pas applicable en présence de nombreux régimes de vagues. Dans cet article, nous y remédions en utilisant un algorithme de classification (K-Moyennes).

La méthode a été appliquée à un site localisé à proximité du Sri Lanka. Les données d'état de mer utilisées proviennent du modèle WaveWatchIII.

\section{Mots-clés :}

Vagues - Statistiques des extrêmes - K-Moyennes - Classification - Océan Indien - Sri Lanka

\footnotetext{
Abstract:

Extreme wave analysis is useful for coastal risk policies. In particular environments such as tropical areas, waves have different origins. In the Indian Ocean for instance, waves can be generated either by austral swells, by monsoon winds and by cyclones. Depending on the meteorological phenomenon that generates waves, the sea state characteristics differ in terms of wave height, wave period and wave direction but they
} 
also differ in terms of return period. The presence of numerous wave regimes complicates extreme wave analysis.

Identifying homogenous wave regimes is a prerequisite for extreme wave analysis. Homogeneous wave regime means a set a wave data that have the same meteorological origin. Usually, homogeneous wave regimes are determined using simple criteria such as the wave direction or the season of occurrence. Unfortunately, such criteria are inadequate in complex wave environments. In this paper, a clustering algorithm is used to identify homogeneous wave regimes. Then, an extreme wave analysis is carried out for each regime.

The method is applied on a site located close to the Sri Lanka. The wave data come from the WaveWatchIII model.

\section{Keywords:}

Wave - Extreme wave analysis - K-Means - Clustering - Indian Ocean - Sri Lanka

\section{Introduction}

La connaissance des états de mer extrêmes est indispensable à la gestion des risques côtiers. Usuellement, le calcul consiste à établir une relation entre la hauteur significative des vagues extrêmes et leur probabilité d'occurrence qui est elle-même reliée à la période de retour (Pr). Pour anticiper toutes les configurations possibles de conditions de vagues extrêmes, il est nécessaire de connaître les hauteurs significatives extrêmes mais également les périodes et les directions associées.

La méthode de détermination des conditions de mer extrêmes nécessite dans un premier temps de constituer des échantillons homogènes d'événements indépendants. Un échantillon homogène d'événements est un nombre fini d'événements ayant des caractéristiques d'état de mer comparables. Typiquement, il s'agit d'événements ayant la même origine météorologique. En pratique, pour avoir des échantillons homogènes, les données de vagues sont séparées par saison et/ou par secteur directionnel (MATHIESEN et al., 1994, MORTON et al., 1997, MENENDEZ et al., 2009). JONATHAN \& EWANS (2007) préfèrent quant à eux intégrer la direction des vagues dans les paramètres d'ajustement des lois statistiques (paramètres qui sont classiquement considérés constants). Cette méthode bien que plus pertinente d'un point de vue mathématique est peu utilisée car elle est assez complexe à mettre en œuvre et fournit des résultats difficiles à interpréter. Dans cette étude, plutôt que d'utiliser des critères basés sur la saisonnalité ou sur la direction de provenance des vagues pour constituer des échantillons homogènes, nous utilisons un algorithme de classification.

Le site d'application est localisé à proximité du Sri Lanka. Ce site a la particularité d'être exposé à des vagues ayant de nombreuses origines. 


\section{Méthodes mathématiques}

\subsection{Classification des vagues}

BUTEL et al. (2002) ont testé des méthodes de classification de données permettant d'obtenir une représentation synthétique d'un ensemble d'observations du signal de houle : le recuit simulé et les méthodes de FORGY (1965) et de BALL and HALL (1967) qui sont des variantes de l'algorithme des K-Moyennes. Ces algorithmes de classification regroupent les observations $\left(\mathrm{H}_{\mathrm{s}}, \mathrm{T}_{\mathrm{p}}, \mathrm{D}_{\mathrm{p}}\right)$ en familles homogènes selon un critère de ressemblance calculé avec la distance euclidienne normalisée entre deux triplets $\left(\mathrm{H}_{\mathrm{s}, 1}, \mathrm{~T}_{\mathrm{p}, 1}, \mathrm{D}_{\mathrm{p}, 1}\right)$ et $\left(\mathrm{H}_{\mathrm{s}, 2}, \mathrm{~T}_{\mathrm{p}, 2}, \mathrm{D}_{\mathrm{p}, 2}\right)$ :

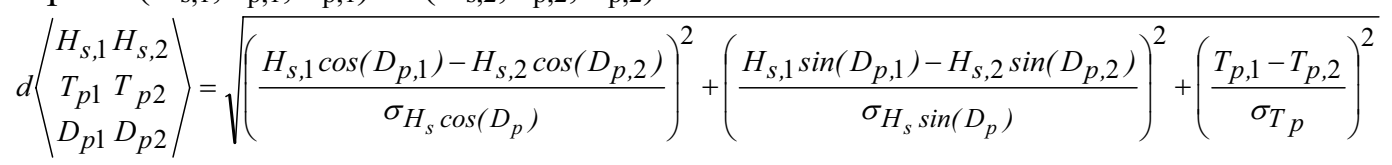

où $\sigma_{\alpha}$ désigne l'écart-type sur le paramètre $\alpha$ qui est calculé sur l'ensemble des données.

Des tests de comparaison entre K-moyennes et recuit simulé ont montré que les résultats obtenus sont très proches. Le temps de calcul du K-Moyennes étant nettement inférieur à celui du recuit simulé, c'est l'algorithme K-Moyennes qui est utilisé dans cet article. L'algorithme K-Moyennes est une méthode itérative qui consiste dans un premier temps à choisir aléatoirement $\mathrm{K}$ centroïdes $\left(\mathrm{H}_{\mathrm{s}, \mathrm{i}}, \mathrm{T}_{\mathrm{p}, \mathrm{i}}, \mathrm{D}_{\mathrm{p}, \mathrm{i}}\right)_{\mathrm{i}=1, \mathrm{~K}}$. Ensuite, chaque triplet est associé au centroïde le plus proche ce qui permet de constituer $\mathrm{K}$ groupes. Les positions des centroïdes dans l'espace à trois dimensions sont alors réévaluées en calculant les centres de gravité des groupes ainsi constitués. Les opérations successives de constitution des groupes - réévaluation de la position des centroïdes sont arrêtées quand la position des centroïdes devient suffisamment stable.

\subsection{Statistiques des extrêmes}

Dans cette étude, l'analyse statistique des extrêmes est réalisée avec un programme MATLAB dans lequel plusieurs procédures issues de la boîte à outils WAFO (Wave Analysis for Fatigue and Oceanography ; http://www.maths.lth.se/matstat/wafo/) ont été intégrées. La méthode utilisée est classique ; les différentes étapes mises en œuvre sont les suivantes: (i) identification d'événements extrêmes indépendants (sélection des événements par la méthode des blocks maxima ou par la méthode des seuils), (ii) ajustement de plusieurs lois candidates et calcul des intervalles de confiance (en première approximation, à l'aide de la méthode delta), (iii) choix de la loi qui donne le meilleur ajustement (le choix porte sur les résultats de tests statistiques (test du $\chi^{2}$ et test de Kolmogorov-Smirnov) et sur le nombre de valeurs situées en dehors des intervalles de confiance) et (iv) sortie des résultats. En fonction de la méthode de sélection des événements indépendants, nous utilisons soit des lois basées sur la probabilité de renouvellement (Weibull, Gumbel, normale, Generalized Extreme Value) soit des lois 
basées sur la probabilité de dépassement d'un seuil (Generalized Pareto Distribution). Différentes méthodes d'ajustement sont testées pour les lois à trois paramètres : le maximum de vraisemblance (ML), la méthode des moments pondérés (PWM), la méthode des moments (MOM) et la méthode de Pickands (PKD).

\section{Caractéristiques des vagues du site}

Les données utilisées sont des sorties du modèle NOAA WaveWatchIII (TOLMAN, 2002). Les caractéristiques des vagues ont été extraites en un point situé à une centaine de kilomètres à l'Est des côtes du Sri Lanka. La coordonnée du point est $\left(8^{\circ} \mathrm{N}\right.$; $82,50^{\circ} \mathrm{E}$ ). La période couverte s'étend du 30/01/1997 au 1/11/2008 (34345 valeurs sont utilisées ; le pas de temps est de $3 \mathrm{~h}$ ).

Les côtes Sri Lankaises sont parfois affectées par des cyclones. Etant donné que ces événements sont rares et que la trajectoire des cyclones est très variable, il est impossible d'estimer les hauteurs de vagues extrêmes générées par les cyclones en utilisant la méthode décrite dans la section 2.2. Pour éviter d'introduire un biais dans le calcul des hauteurs de vagues extrêmes, nous avons décidé d'extraire les données associées aux vagues cycloniques. Pour chaque événement indépendant identifié lors de l'étape (i) de l'analyse des extrêmes (cf. section 2.2), nous avons regardé s'il y avait une activité cyclonique à proximité de la zone d'étude. La base de données du Joint Typhoon Warning Center (JTWC) a été utilisée pour cela. A l'issue de cette étape, seules les données qui correspondent au cyclone du 25 décembre 2000 ont été retirées. Les vagues générées lors de ce cyclone sont les plus grosses qui ont été atteintes pendant la période d'étude $\left(\mathrm{H}_{\mathrm{s}}=4,3 \mathrm{~m}\right)$. Une méthode spécifique doit absolument être mise en œuvre pour les vagues cycloniques (CHU \& WANG, 1998, PHADKE et al., 2003).

\section{Identification des régimes de vagues homogènes}

Afin d'identifier des ensembles homogènes de données de vagues, les graphes bivariés $T_{p}\left(H_{s}\right)$ d'une part et $D_{p}\left(H_{s}\right)$ d'autre part sont tracés sur la figure 1. Cette figure fait apparaître plusieurs nuages de points ce qui révèle la diversité d'origine des vagues. En termes de période, une distinction apparaît entre les périodes courtes qui correspondent aux vagues de moussons et les périodes longues qui correspondent aux houles australes. Globalement, trois nuages de points se distinguent en termes de direction de provenance : les directions comprises entre $0^{\circ} \mathrm{N}$ et $120^{\circ} / \mathrm{N}$ correspondent aux vagues de mousson d'hiver; les directions comprises entre 110 et $140^{\circ} / \mathrm{N}$ correspondent aux houles australes. Les vagues de mousson d'été ont des directions qui varient fortement autour d'une valeur proche de $250^{\circ} / \mathrm{N}$.

Pour les valeurs élevées de $\mathrm{H}_{\mathrm{s}}\left(\mathrm{H}_{\mathrm{s}}>2 \mathrm{~m}\right)$, il est impossible de déterminer l'origine des vagues sur la base d'un critère de direction seulement. Il existe en effet une ambiguïté lorsque les secteurs directionnels associés aux trois régimes identifiés se chevauchent. 
Par exemple, pour les valeurs de $\mathrm{D}_{\mathrm{p}}$ de l'ordre de $200^{\circ} / \mathrm{N}$, les vagues de mousson d'été se confondent avec les houles australes. Un algorithme de classification est utilisé pour essayer de palier à cette limitation. Cette méthode à en effet l'avantage d'utiliser plusieurs critères pour constituer des ensembles d'état de mer homogènes.
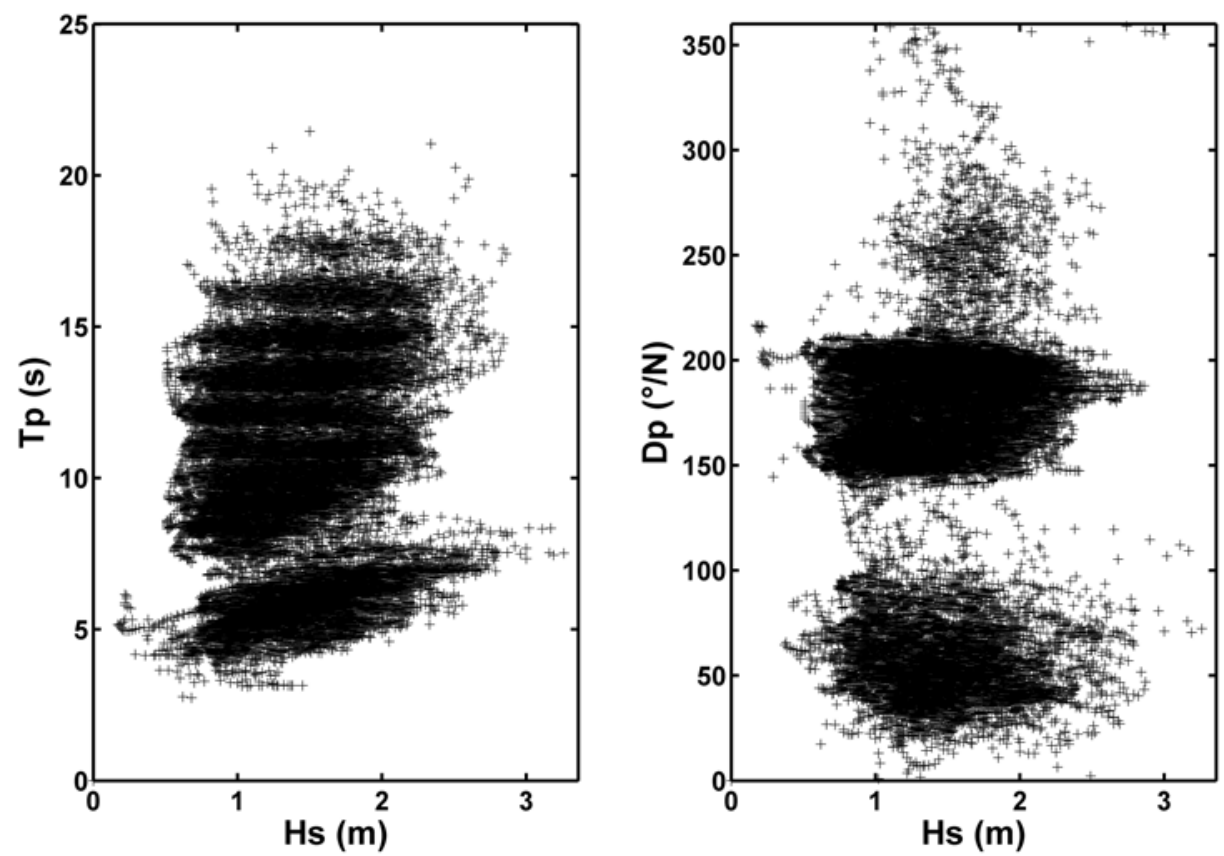

Figure 1. A gauche) Graphe bivarié représentant la période de pic en fonction de la hauteur significative des vagues. A droite) Graphe bivarié représentant la direction de pic en fonction de la hauteur significative des vagues.

L'algorithme K-Moyennes nécessite de choisir a priori un nombre K de classes. Après plusieurs tests, $\mathrm{K}$ a été fixé à 15 . Des valeurs plus faibles de $\mathrm{K}$ ne permettaient pas d'isoler correctement les modes de mousson d'hiver et d'été. Les résultats de la classification sont présentés dans le tableau 1. Les 15 classes ont été regroupées en 3 régimes principaux : (i) les houles australes qui représentent $70 \%$ des observations sont caractérisées en particulier par de longues périodes et par une direction de provenance du Sud, (ii) les vagues de mousson d'hiver (24\% des observations) sont caractérisées par des petites périodes et proviennent du Nord-Est, (iii) les vagues de mousson d'été ( $6 \%$ des observations) ont de petites périodes et proviennent du Sud-Ouest.

La saisonnalité des régimes identifiés montre une prédominance des houles australes en été (figure 2). Cette figure montre également qu'il aurait été impossible de constituer des ensembles homogènes d'états de mer sur la base d'un critère de saisonnalité. En effet, pendant l'été, il y a aussi bien des vagues australes que des vagues de mousson d'été. 
Tableau 1. Caractéristiques des classes de vagues. Hiver réfêre à la période de décembre à février. Les directions $0 \% / N$ indiquent des vagues en provenance du Nord et $90^{\circ} / \mathrm{N}$ des vagues provenant de l'Est.

\begin{tabular}{lllll}
\hline Classes de vagues & $\boldsymbol{H}_{\boldsymbol{s}}(\boldsymbol{m})$ & $\boldsymbol{T}_{\boldsymbol{p}}(\mathbf{s})$ & $\boldsymbol{D}_{\boldsymbol{p}}(\mathbf{N})$ & Occurrence (\%) \\
\hline Mousson été & 1,57 & 5,2 & 257 & 2,6 \\
Mousson été & 1,59 & 6,1 & 202 & 2,9 \\
Mousson hiver & 1,06 & 5,4 & 69 & 7,5 \\
Mousson hiver & 1,28 & 5,8 & 37 & 6,7 \\
Mousson hiver & 1,81 & 6,4 & 48 & 6,3 \\
Mousson hiver & 1,81 & 6,6 & 75 & 3,7 \\
Houle australe, annuel, surtout été & 1,48 & 9,6 & 155 & 9,0 \\
Houle australe, annuel, surtout printemps, automne & 0,99 & 8,6 & 156 & 10,0 \\
Houle australe, annuel, surtout printemps, automne & 1,05 & 12,6 & 196 & 10,6 \\
Houle australe, annuel, surtout printemps, automne & 1,07 & 10,4 & 176 & 7,1 \\
Houle australe, toute l'année & 1,19 & 15,1 & 195 & 5,5 \\
Houle australe, été, automne & 1,68 & 13,8 & 198 & 10,2 \\
Houle australe, été & 1,74 & 12,2 & 186 & 6,1 \\
Houle australe, été & 1,76 & 10,6 & 169 & 6,5 \\
Houle australe, été & 1,92 & 16,2 & 192 & 5,3 \\
\hline
\end{tabular}

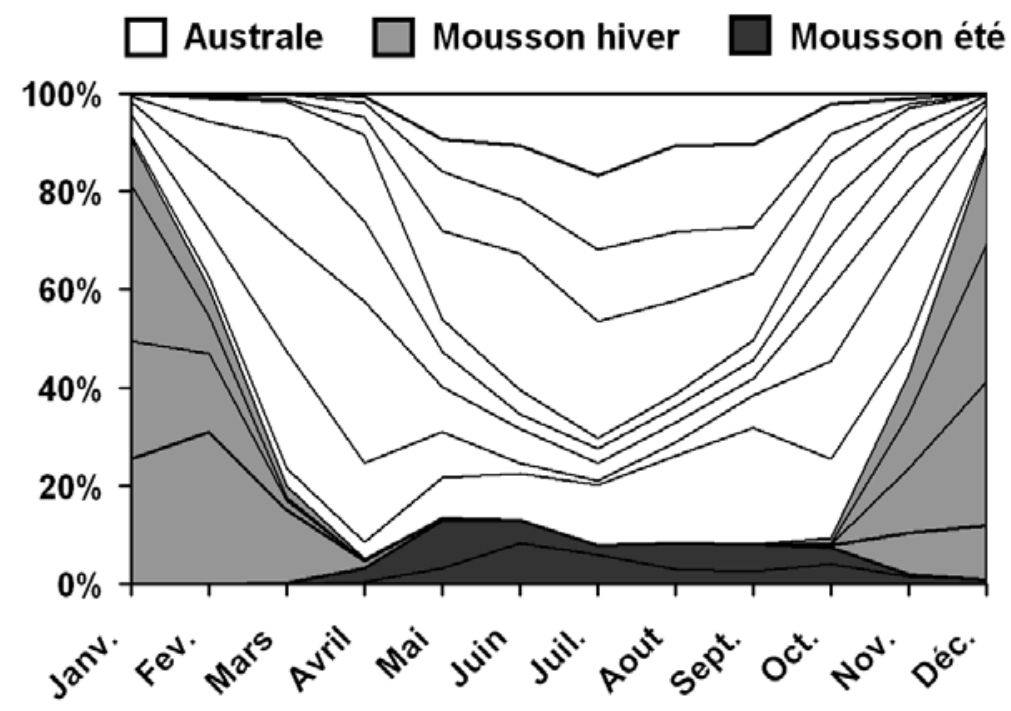

Figure 2. Saisonnalité des régimes de vagues identifiés à l'aide de la classification.

\section{Statistiques des extrêmes pour chaque régime de vagues}

Les hauteurs significatives extrêmes sont maintenant analysées statistiquement en utilisant les trois ensembles d'états de mer homogènes constitués à partir des résultats de la classification. Pour les trois analyses (houles australes, vagues de mousson d'été et 


\section{XI $I^{\text {èes }}$ Journées Nationales Génie Côtier - Génie Civil}

Les Sables d'Olonne, 22-25 juin 2010

vagues de mousson d'hiver), c'est la loi GPD qui est retenue. Les résultats sont représentés sur les figures 3,4 et 5 et dans le tableau 2 .

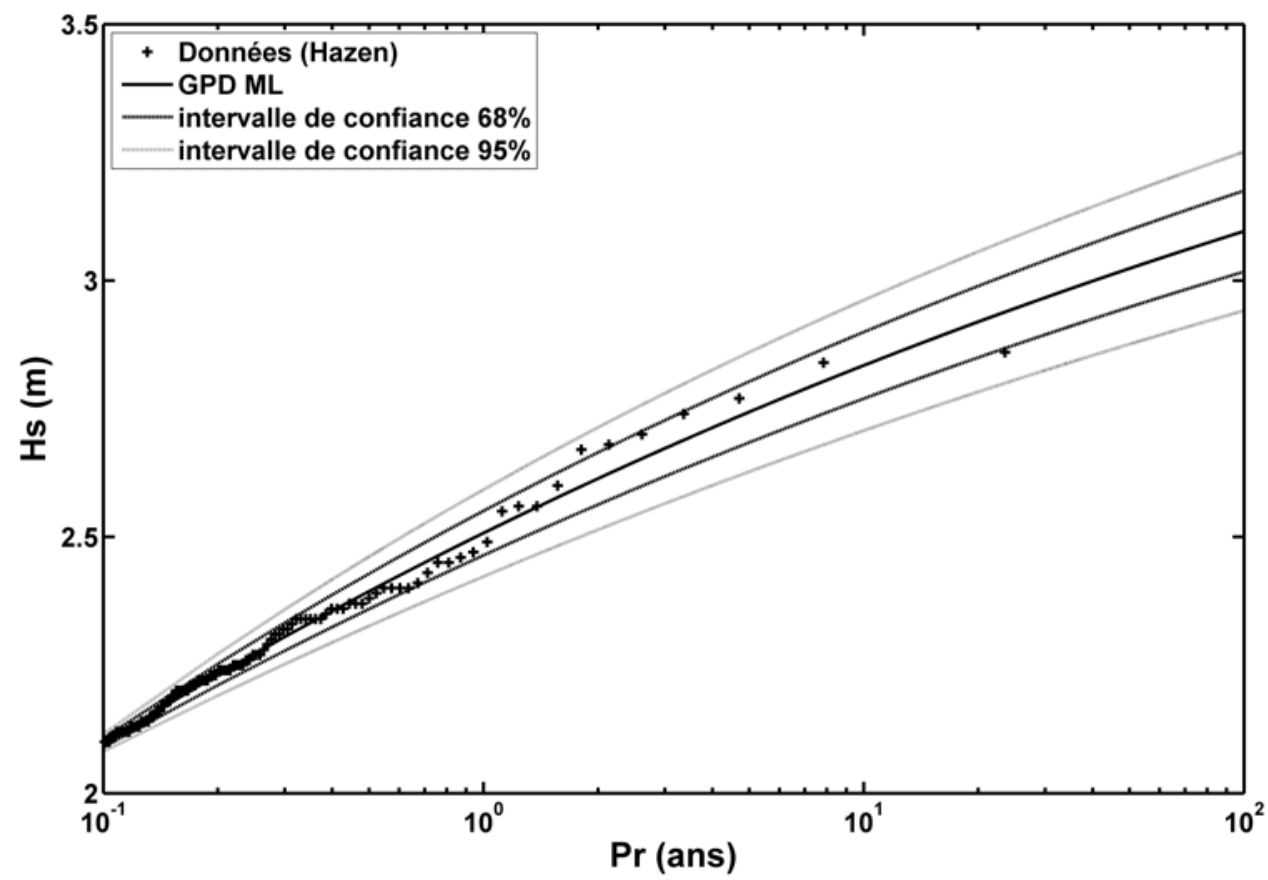

Figure 3. Houles australes; hauteur significative des vagues en fonction de la période de retour.

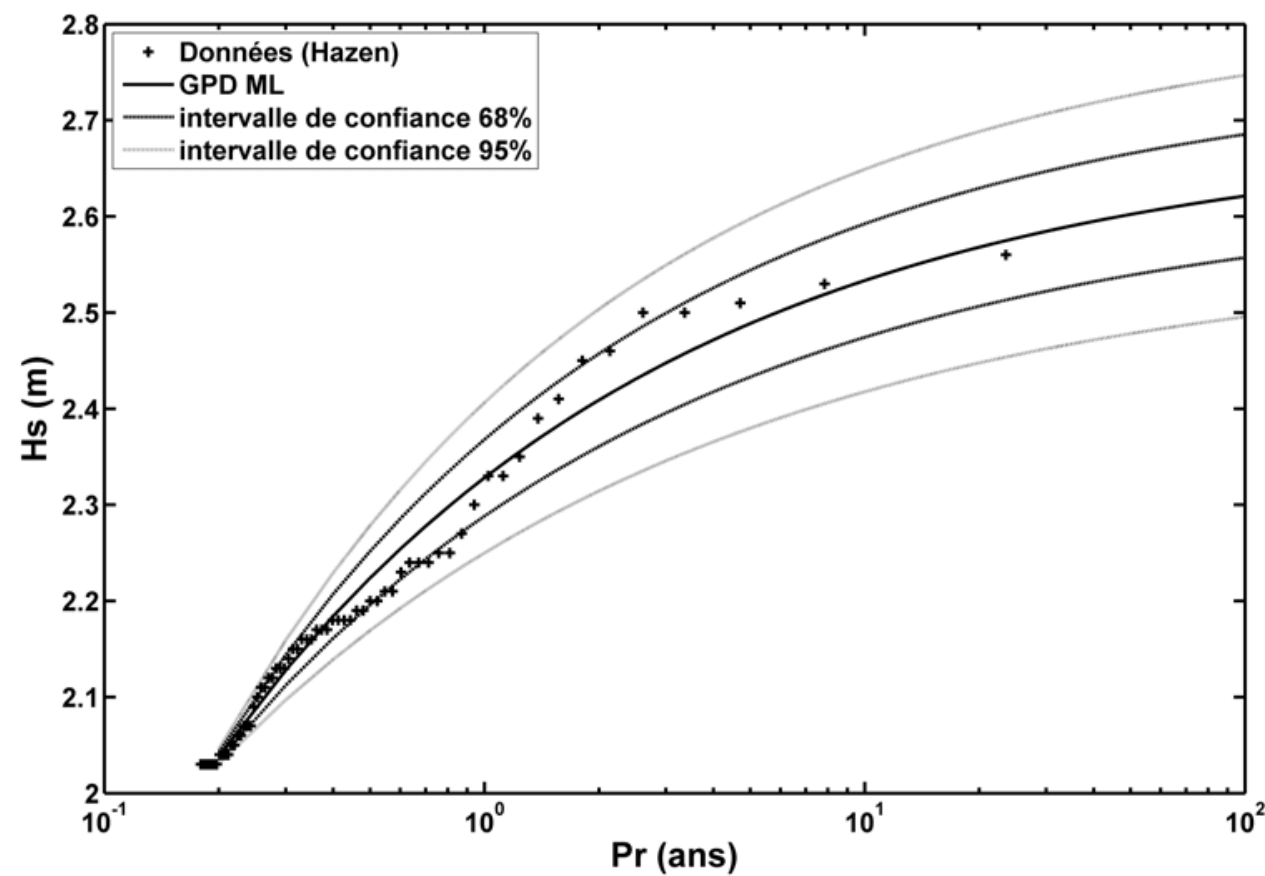

Figure 4. Vagues de mousson d'été ; hauteur significative des vagues en fonction de la période de retour. 


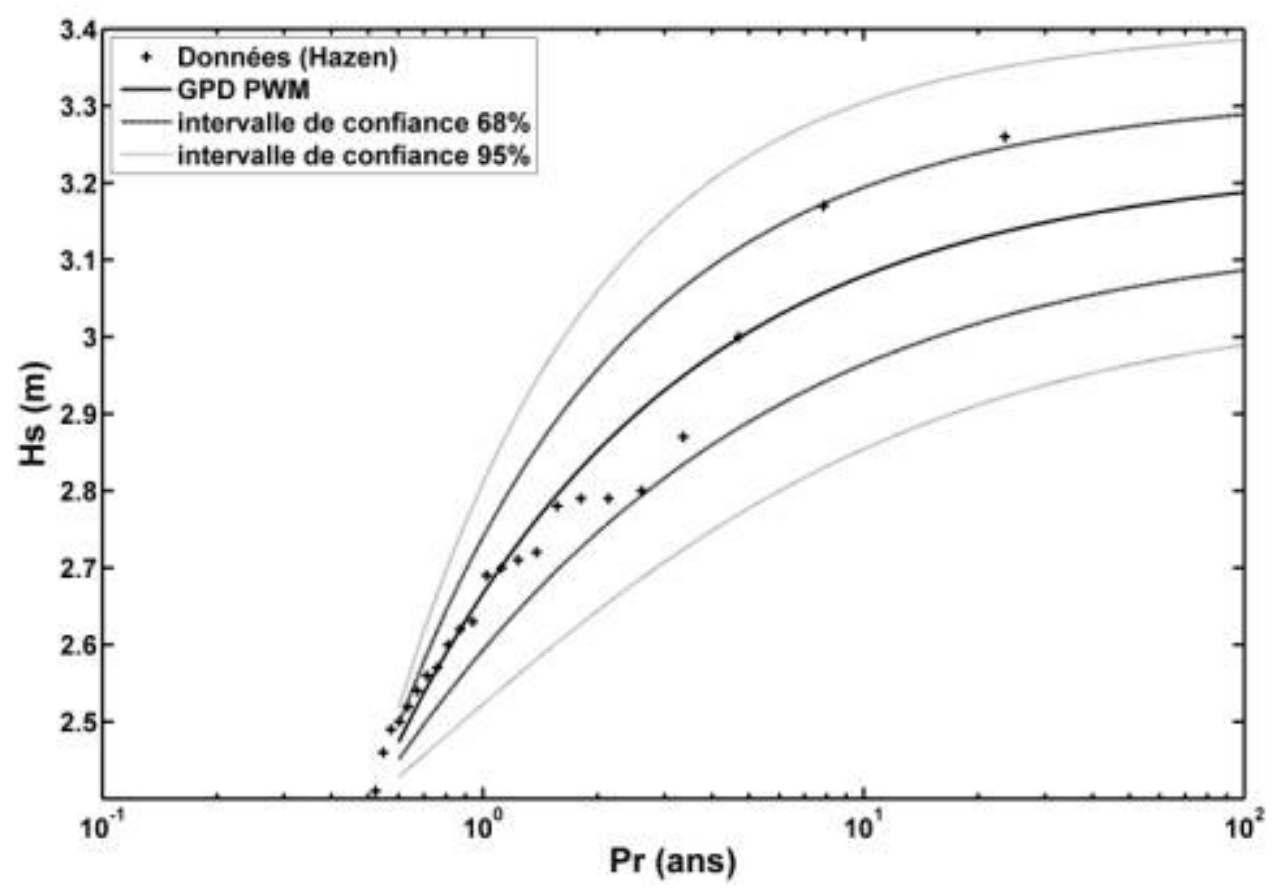

Figure 5. Vagues de mousson d'hiver; hauteur significative des vagues en fonction de la période de retour.

Une fois les hauteurs de vagues significatives extrêmes estimées, deux approches sont envisagées pour déterminer les périodes et les directions associées aux événements extrêmes de chaque régime. La première, notée $\mathrm{A} 1$, consiste à estimer la période et la direction des vagues extrêmes en utilisant les valeurs de $T_{p}$ et de $D_{p}$ correspondant à la classe la plus énergétique du régime considéré (i.e. celle qui a la hauteur $\mathrm{H}_{\mathrm{s}}$ la plus élevée, voir tableau 1). Ainsi, pour la houle australe par exemple, les valeurs de période et de direction au pic du spectre pendant les événements extrêmes sont estimées à $16,2 \mathrm{~s}$ et $192^{\circ} / \mathrm{N}$ respectivement. Les valeurs pour les autres régimes sont fournies dans le tableau 2. L'approche A1 permet d'estimer rapidement la direction de pic et la période de pic des états de mer extrêmes mais elle est à considérer avec précaution lorsque la hauteur $\mathrm{H}_{\mathrm{s}}$ à laquelle on cherche à associer une période et une direction diffère significativement de la hauteur $\mathrm{H}_{\mathrm{s}}$ de classe la plus énergétique. Dans ce cas, la deuxième approche notée A2 pourra être utilisée. A2 consiste à analyser les graphes bivariés associés à chaque régime (voir figure 6). Ces graphes sont en effet assez denses pour pouvoir établir, par régression, une loi simple reliant les variables $T_{p}$ et $H_{s}$ d'une part et $\mathrm{D}_{\mathrm{p}}$ et $\mathrm{H}_{\mathrm{s}}$ d'autre part. Pour une première analyse, des régressions linéaires ont été utilisées (seules les valeurs supérieures à $2 \mathrm{~m}$ ont été considérées afin de focaliser sur le comportement extrême du système). Les résultats sont synthétisés dans le tableau 2 . Les écarts - types en termes de périodes de pic et de directions de pic valent 2,2s, 0,6s et $0,5 \mathrm{~s}$ et $13^{\circ} / \mathrm{N}, 32^{\circ} / \mathrm{N}$ et $19^{\circ} / \mathrm{N}$ pour les houles australes, les vagues de mousson d'été et d'hiver respectivement. 

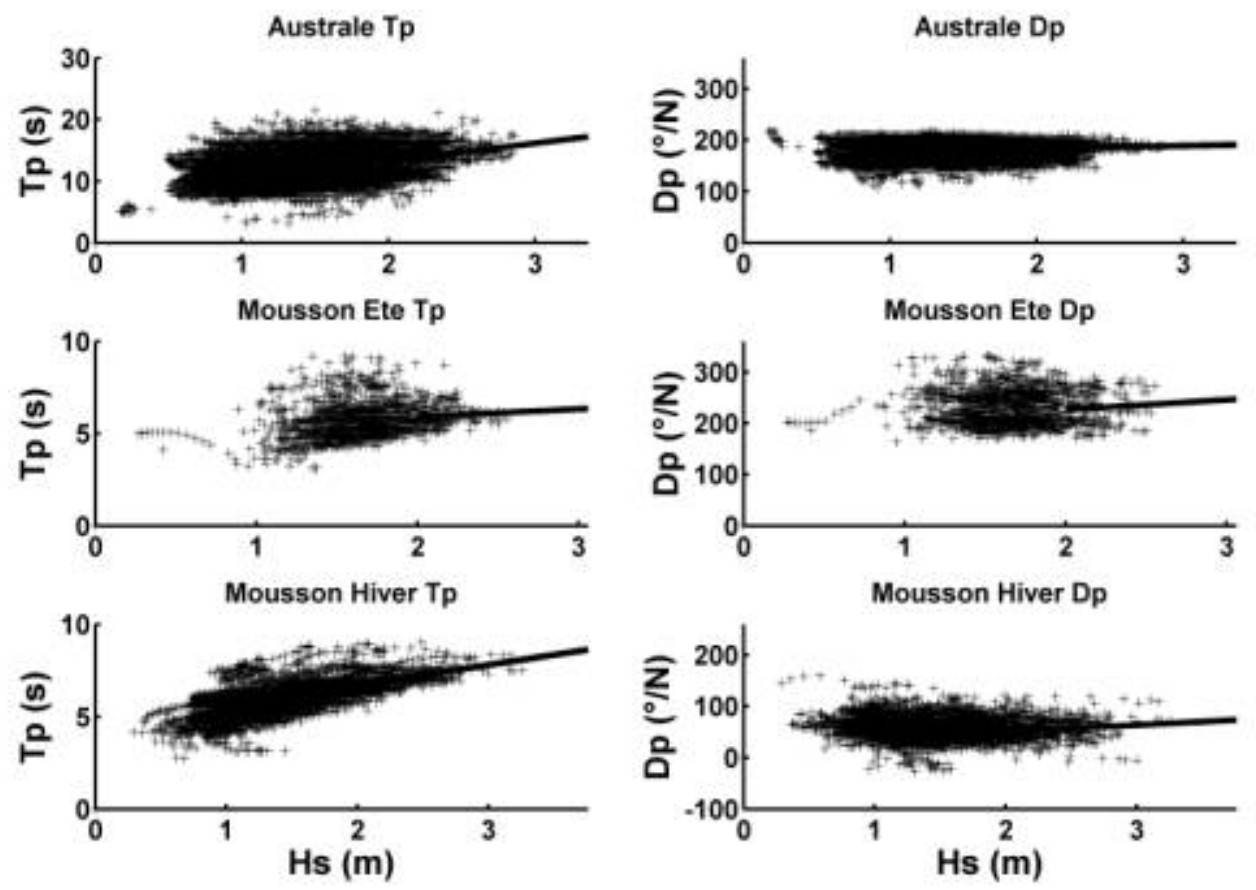

Figure 6. Graphes bivariés représentant la période de pic des vagues en fonction de la hauteur significative des vagues (à gauche), la direction de pic des vagues en fonction de la hauteur significative des vagues (à droite).

Tableau 2. Caractéristiques des vagues pour des périodes de retour de 1, 10 et 50 ans.

\begin{tabular}{|c|c|c|c|c|}
\hline & $P r$ & 1 an & 10 ans & 50 ans \\
\hline \multirow[t]{3}{*}{ Australe } & $\left(H_{s} ; T_{p} ; D_{p}\right)-A 1$ & $(2,51 ; 16,2 ; 192)$ & $(2,85 ; 16,2 ; 192)$ & $(2,96 ; 16,2 ; 192)$ \\
\hline & $\left(H_{s} ; T_{p} ; D_{p}\right)-A 2$ & $(2,51 ; 14,7 ; 186)$ & $(2,85 ; 15,7 ; 188)$ & $(2,96 ; 16 ; 189)$ \\
\hline & IC à $68 \%$ pour $H_{s}$ & {$[2,44 ; 2,57]$} & {$[2,62 ; 3,08]$} & {$[2,71 ; 3,22]$} \\
\hline Mousson & $\left(H_{s} ; T_{p} ; D_{p}\right)-A 1$ & $(2,31 ; 6,1 ; 202)$ & $(2,55 ; 6,1 ; 202)$ & $(2,57 ; 6,1 ; 202)$ \\
\hline \multirow[t]{2}{*}{ été } & $\left(H_{s} ; T_{p} ; D_{p}\right)-A 2$ & $(2,31 ; 6 ; 232)$ & $(2,55 ; 6,1 ; 237)$ & $(2,57 ; 6,1 ; 238)$ \\
\hline & IC à $68 \%$ pour $H_{s}$ & {$[2,26 ; 2,36]$} & {$[2,39 ; 2,71]$} & {$[2,42 ; 2,71]$} \\
\hline Mousson & $\left(H_{s} ; T_{p} ; D_{p}\right)-A 1$ & $(2,66 ; 6,5 ; 61,5)$ & $(3,13 ; 6,5 ; 61,5)$ & $(3,27 ; 6,5 ; 61,5)$ \\
\hline \multirow[t]{2}{*}{ hiver } & $\left(H_{s} ; T_{p} ; D_{p}\right)-A 2$ & $(2,66 ; 7,4 ; 59)$ & $(3,13 ; 7,9 ; 65)$ & $(3,27 ; 8,1 ; 67)$ \\
\hline & IC à $68 \%$ pour $H_{s}$ & {$[2,52 ; 2,79]$} & {$[2,91 ; 3,35]$} & {$[3,05 ; 3,49]$} \\
\hline
\end{tabular}

\section{Conclusion}

Dans les environnements aux caractéristiques météorologiques complexes tels que les zones tropicales, il existe plusieurs sources d'états de mer extrêmes et il est par conséquent délicat d'identifier des ensembles homogènes de données de vagues. Cette étape est pourtant nécessaire pour pouvoir effectuer des analyses statistiques d'états de mer extrêmes. Bien que la classification des vagues soit classiquement utilisée pour analyser les caractéristiques des vagues moyennes, son utilisation s'est avérée adaptée pour identifier des ensembles homogènes de données de vagues extrêmes. 
Pour le site étudié, l'analyse des conditions extrêmes pour chaque régime de vague montre que les conditions les plus extrêmes sont liées aux vagues de mousson d'hiver. La hauteur significative décennale pour ce type de vague est de 3,1 m. La direction de provenance associée à ce type d'état de mer est Ouest-Nord-Ouest. Les périodes de pic sont de l'ordre de $8 \mathrm{~s}$. Les houles australes qui proviennent du Sud ont une hauteur $\mathrm{H}_{\mathrm{s}}$ décennale de l'ordre de 2,9 m. Elles sont caractérisées par des périodes de pic longues (16 s pour l'état de mer décennale). Enfin les vagues de mousson d'été ont des directions de provenance qui varient fortement autour du Sud-Ouest. Les hauteurs significatives et les périodes de pic associées aux états de mer décennaux sont 2,6 m et 6 s respectivement.

\section{Références bibliographiques}

BALL G.H., HALL D.J. (1967) A clustering technique for summarizing multivariate data. Behavior Science 12, 153-155. doi:10.1002/bs.3830120210

BUTEL R., DUPUIS H., BONNETON P. (2002). Spatial Variability of Wave Conditions on the French Atlantic Coast using In-Situ Data. Journal of Coastal Research, SI 36, pp 96-108.

CHU P.S., WANG J.X. (1998). Modeling return periods of tropical cyclone intensities in the vicinity of Hawaii. Journal of Applied. Meteorology, 37, pp 951-960. doi:10.1175/1520-0450(1998)037<0951:MRPOTC $>2.0 . C O ; 2$

FORGY E.W. (1965) Cluster analysis of multivariate data: efficiency versus interpretability of classifications. Paper presented at biometric society meetings, Riverside, California, abstracts in Biometrics, 21, 3.

JONATHAN P., EWANS K. (2007). The effect of directionality on extreme wave design criteria. Ocean Engineering, 34, 14-15, pp 1977-1994.

MATHIESEN M., GODA Y., HAWKES P.J., MANSARD E., MARTÍN M.J., PELTIER E., THOMPSON E.F., VAN VLEDDER G. (1994). Recommended practice for extreme wave analysis. Journal of Hydraulic Research, 32, 6.

MENENDEZ M., MENDEZ F.J., IZAGUIRRE C., LUCENO A., LOSADA I.J. (2009). The influence of seasonality on estimating return values of significant wave height. Coastal Engineering, 56, 3, pp 211-219. doi:10.1016/j.coastaleng.2008.07.004

MORTON I.D., BOWERS J., MOULD G. (1997). Estimating return period wave heights and wind speeds using a seasonal point process model. Coastal Engineering, 31, 1-4, pp 305-326. doi:10.1016/S0378-3839(97)00016-1

PHADKE A.C., MARTINO C.D., CHEUNG K.F., HOUSTON S.H. (2003) Modeling of tropical cyclone winds and waves for emergency management. Ocean Engineering, 30, 4, pp 553-578. doi:10.1016/S0029-8018(02)00033-1

TOLMAN H.L. (2002). User manual and system documentation of WAVEWATCH-III version 2.22. NOAA / NWS / NCEP / OMB. Technical note 222, $133 \mathrm{p}$. 